\title{
Consommations énergétiques et coût d'exploitation des procédés de concentration
}

\author{
par \\ J. HUCHON et J.F. REYNAUD
}

Le but de l'opération de concentration est d'accroître la teneur en matières sèches (MS) des produits traités (laits, lactosérum, babeurre...) dans des proportions variables selon la fabrication concernée.

Le terme concentration recouvre, dans la réalité, une variété d'opérations que l'on peut différencier d'après la valeur de la teneur en matières sèches atteinte à la fin de ces opérations. Les valeurs maximales sont variables en fonction du devenir des produits:

lactosérum $32 \%$ MS avant transport,

lait écrémé $48-50 \%$ MS avant atomisation, etc.

Selon la concentration finale, on distingue trois opérations :

- la concentration se limite à :

18 à $38 \%$ MS avec le lait écrémé

12 à $32 \%$ MS avec le lactosérum

- la concentration proprement dite permet d'atteindre :

48 à $50 \%$ MS avec le lait écrémé

50 à $55 \%$ MS avec le lactosérum

- la finition consiste à transformer un préconcentré dans un concentrateur appelé finisseur. Elle permet d'atteindre des taux de matières sèches très élevés :

$60 \%$ et plus sur le lactosérum.

L'augmentation de la teneur en matières sèches peut être obtenue en éliminant l'eau soit par voie physique, osmose inverse, soit par voie thermique, évaporation.

Les procédés classiques d'évaporation consomment de la vapeur produite à partir de combustible fossile. Or, depuis quelques années, se confirme le développement important de procédés utilisant

Ampère 82, Tour Atlantique, 9, place de la Pyramide - 92080 Paris - La Défense cedex 6 . 
l'énergie électrique : évaporation avec recompression mécanique de vapeur et osmose inverse.

Nous ne développerons ici que l'osmose inverse, thème retenu pour la présente journée de travail.

\section{CONCENTRATION PAR OSMOSE INVERSE}

\section{Rappels}

La séparation d'un soluté et d'un solvant par passage de ce dernier, sous l'effet d'une pression, à travers une membrane semiperméable peut se faire par ultrafiltration ou par osmose inverse.

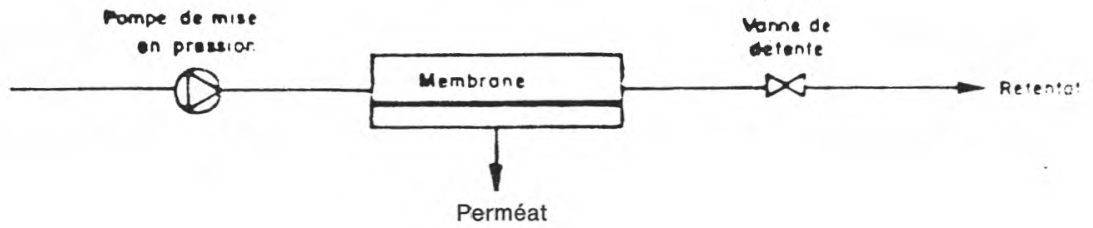

fig. 1

Schéma de principe.

L'ultrafiltration (UF) a pour but de retenir les grosses molécules, le perméat étant constitué d'une partie du solvant (souvent de l'eau) et des petites molécules. La pression appliquée est faible (moins de 10 bars).

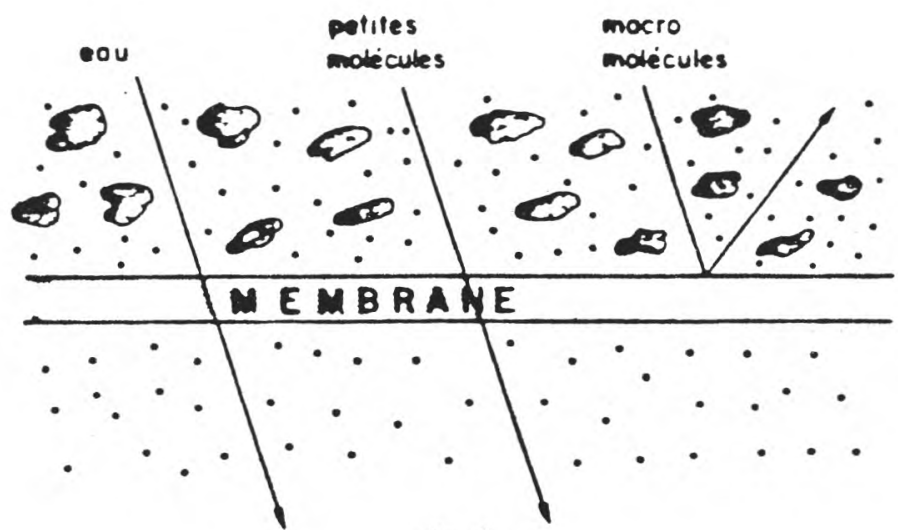

fig. 2

L'ultrafiltration. 
La rétention des grosses molécules s'explique en grande partie par un effet de tamisage de celles-ci dans les pores de la membrane en fonction des dimensions respectives des unes et des autres.

L'osmose inverse (OI) laisse passer l'eau et retient la plupart des molécules dissoutes, même petites. La pression appliquée est élevée (jusqu'à 80 bars) du fait de la grande différence de pression osmotique entre rétentat et perméat.

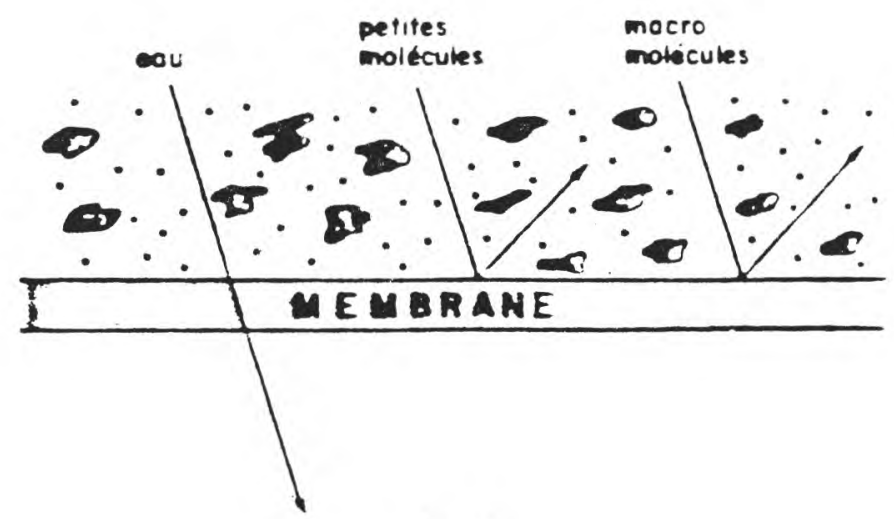

fig. 3

L'osmose inverse.

La taille des solutés retenus étant peu différente de celle du solvant, on doit avant tout faire appel aux interactions physicochimiques entre membrane et constituants de la solution pour expliquer la semi-perméabilité.

Ainsi, si l'ultrafiltration s'avère adaptée à la séparation et à la purification des protéines, seule l'osmose inverse, qui retient toutes les molécules du soluté, permet la concentration d'une solution au sens strict du terme.

\section{Technologie}

\subsection{Membranes}

La conception d'une membrane se heurte à plusieurs exigences contradictoires :

- réaliser la séparation, ce qui suppose une structure relativement fermée ;

- avoir une bonne résistance mécanique, ce qui implique une épaisseur suffisante ; 
- assurer un débit de perméat compatible avec les exigences industrielles; or le débit est d'autant plus grand que la structure est ouverte et l'épaisseur faible (loi de Poiseuille).

Ce problème a été résolu par la mise au point de membranes asymétriques constituées d'une " peau » superficielle très fine qui lui confère ses caractéristiques de semi-perméabilité sans perte de charge excessive et d'un support poreux n'ayant aucun rôle dans la séparation mais qui assure les caractéristiques mécaniques recherchées (cf fig. 4).

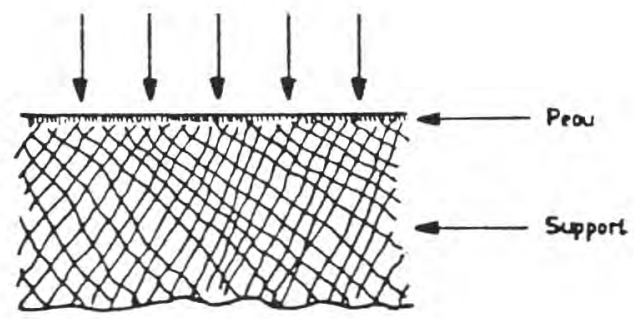

Membrene esymétrique

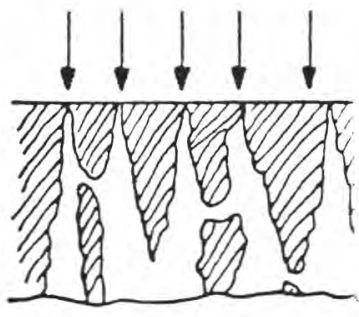

Schéme des pores de la membrone osjmetrique

fig. 4

Schéma de la structure d'une membrane.

Jusqu'en 1981, les membranes d'osmose inverse étaient en acétate de cetlulose. Il en résultait certaines contraintes d'utilisation :

- la température ne devait pas dépasser $35^{\circ} \mathrm{C}$;

- le $\mathrm{pH}$ devait être situé entre 3 et 8 .

Ces contraintes éatient difficilement compatibles avec le traitement de certains liquides alimentaires. De plus, les débits de perméat croissent avec la température et l'efficacité des produits de nettoyage est plus importante aux $\mathrm{pH}$ extrêmes.

Depuis 1982, les constructeurs proposent des membranes en polymères de synthèse dont les conditions d'utilisation sont les suivantes : $60^{\circ} \mathrm{C}$;

- la température moyenne de traitement ne doit pas dépasser

- le $\mathrm{pH}$ doit être situé entre 2 et 11 .

Ces améliorations significatives des contraintes de fonctionnement ont permis d'élargir le champ d'application de l'osmose inverse dont un des handicaps reste le taux de concentration maximale (28\% MS pour le lactosérum par exemple). 


\subsection{Modules}

Les modules d'osmose inverse utilisés industriellement en 1983 sont de deux types :

- Les modules tubulaires (type PCI)

La membrane est coulée ou rapportée à l'intérieur d'un tube support de grande longueur et de quelques centimètres de diamètre.

Le liquide à traiter circule à vitesse élevée (plusieurs mètres par seconde), en régime turbulent, à l'intérieur du tube; le perméat est récupéré à l'extérieur.

- Les modules plans (type DDS)

Cette technologie est analogue à celle des filtres-presses. Le liquide à traiter circule à vitesse élevée au contact de membranes planes circulaires supportées par des plaques porte-membranes à l'intérieur desquelles le perméat est drainé.

\subsection{CONCEPTION D'UNE INSTALLATION}

Les procédés discontinus ne sont quasiment plus utilisés dans l'industrie laitière.

\subsubsection{Procédé continu à passage direct}

Certaines installations qui concentrent le lactosérum de 6 à $12 \%$ MS sont conçues sur le principe de la concentration continue à passage direct (cf fig. 5).

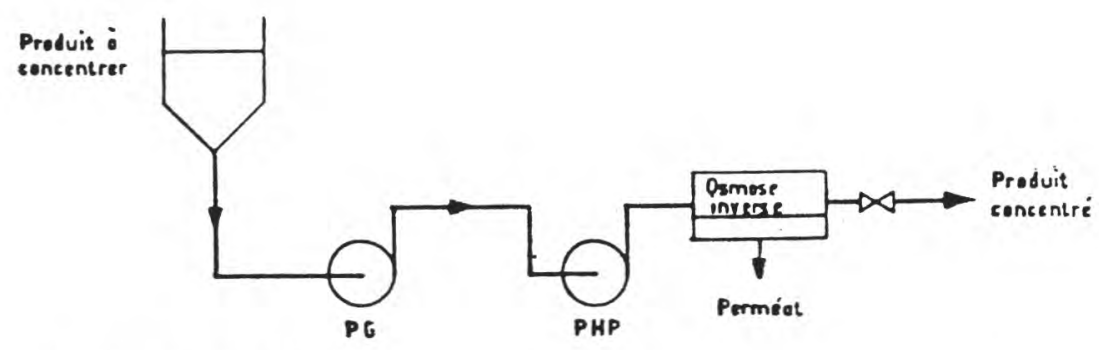

PG : pompe de govege

PKP: pompe houte pression.

fig. 5

Concentration par osmose inverse à passage direct.

\subsubsection{Procédé continu avec recirculation}

Le procédé de traitement le plus utilisé industriellement est la concentration continue avec recirculation (cf fig. 6). 


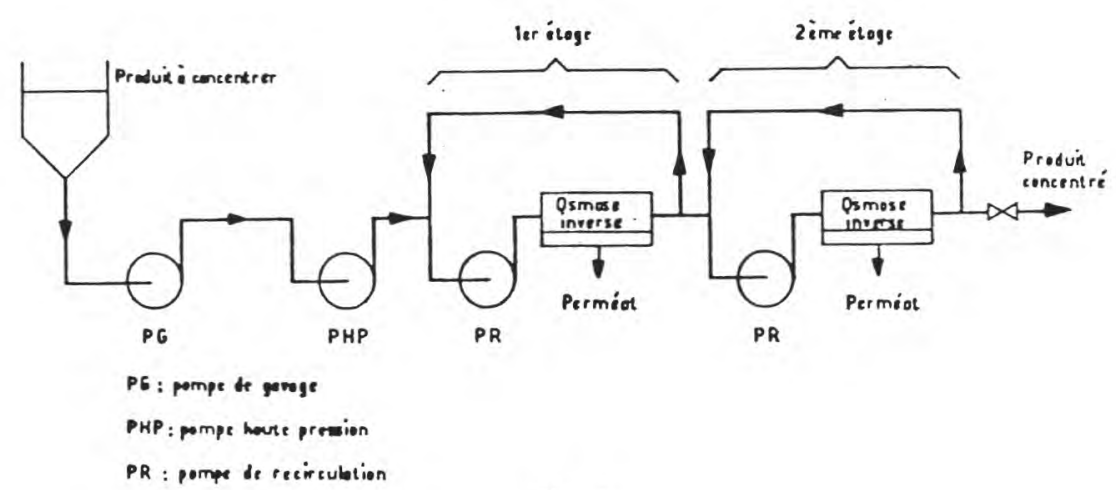

fig. 6

Concentration par osmose inverse avec recirculation (2 étages).

L'installation peut comprendre plusieurs boucles de recirculation (ou étages). Chaque étage est constitué de plusieurs modules et d'une pompe de recirculation.

\section{Coût d'exploitation}

\subsection{ENERGIE}

Les consommations énergétiques moyennes données dans ce paragraphe sont issues des devis présentés par les constructeurs pour des projets étudiés de 1980 à 1983.

Même si dans certains cas un appoint de vapeur est nécessaire pour le chauffage des produits de nettoyage, cette consommation de combustible fossile est négligeable (cf tab. 1).

La consommation énergétique se résume donc uniquement à la consommation d'électricité qui peut varier de 5 à $8 \mathrm{kWh}$ par tonne de perméat c'est-à-dire d'eau éliminée. Soit, à raison de 2,5 thermies par $\mathrm{kWh}$, une consommation spécifique maximale d'énergie primaire de :

$$
2.10^{-3} \text { tep par tonne d'eau éliminée. }
$$

Notons que, pour une installation de concentration par osmose inverse, le coût énergétique représente environ le quart du coût total d'exploitation hors main d'œuvre.

\subsection{COMPARAISON DES COUTS ÉNERGÉTIQUES}

\subsubsection{Coefficient de substitution :}

Si un procédé électrique est en concurrence avec un procédé thermique, la comparaison énergétique des deux solutions est 
exprimée par le coefficient de substitution $\gamma$ qui indique, pour un même service rendu final, combien de thermies utilisées dans une installation consommant un combustible fossile, sont remplacées par $1 \mathrm{kWh}$ dans une installation électrique.

De façon pratique, la définition du coefficient $\gamma$ est un peu plus élaborée du fait qu'une unité ne consomme presque jamais uniquement de la vapeur ou uniquement de l'électricité. Si un procédé « thermique » utilise $\mathrm{Q}_{1}$ thermies vapeur et $\mathrm{W}_{1} \mathrm{kWh}$ et si un procédé " électrique » utilise $\mathrm{Q}_{2}$ thermies vapeur et $\mathrm{W}_{2} \mathrm{kWh}$ (avec $\mathrm{Q}_{1}$ » $\mathrm{Q}_{2}$ et $\mathrm{W}_{1} \ll \mathrm{W}_{2}$ ) le coefficient de substitution sera :

$$
\gamma=\frac{\mathrm{Q}_{1}-\mathrm{Q}_{2}}{\mathrm{~W}_{2}-\mathrm{W}_{1}}(\mathrm{th} / \mathrm{kWh})
$$

Le coefficient de substitution noté $\gamma$ est déterminé au niveau du processus (par exemple l'évaporateur). Au niveau de l'usine, le coefficient de substitution est noté $\gamma_{\mathrm{u}}$ et prend en compte le rendement de production et de distribution de la vapeur.

Ce coefficient permet de mettre en évidence une économie d'énergie primaire chaque fois que $\int$ u est supérieur à 2,5 puisque, par convention en 1984, il faut 2,5 thermies pour produire $1 \mathrm{kWh}$ dans une centrale thermique à combustible fossile et le transporter jusqu'à l'utilisation.

\subsubsection{Indicateur de rentabilité :}

Si le coût moyen du kWh est dans un rapport $\tau$ par comparaison avec le coût moyen de la thermie utile obtenue à partir de combustibles fossiles, l'indicateur $\Gamma_{\mathrm{u}} / \tau$ permet de juger approximativement de la rentabilité économique d'une technique électrique vis-à-vis d'une installation consommant de la vapeur sous chaudière, et rendant le même service (pour autant que les autres composantes du coût d'exploitation soient similaires).

Par exemple, dans les conditions économiques de mi-1983, soit $110 \mathrm{~F}$ la tonne de vapeur et $0,30 \mathrm{~F}$ le $\mathrm{kWh}$ :

- le coût moyen de la thermie utile est de : 110/740 $=0,15 \mathrm{~F} /$ th (cf tableaux pages 13 et 15);

- le rapport des coûts énergétiques $\tau$ est de : $0,30 / 0,15$ $=2 \mathrm{th} / \mathrm{kWh}$.

Nous voyons que l'exploitation de la technique électrique sera plus économique que l'installation classique si le $\lceil$ u est supérieur à $2 \mathrm{th} / \mathrm{kWh}$.

\subsection{NetToyage}

Les membranes synthétiques autorisent l'emploi de produits de nettoyage de faibles coûts.

La durée du nettoyage quotidien est de 2 à 3 h. 
La consommation d'électricité est quasiment la même qu'en exploitation normale.

\subsection{MAIN D'EUVRE}

Ces installations, fortement automatisées, ne nécessitent qu'une surveillance réduite. Le coût de main d'œuvre est sensiblement le même que celui d'un évaporateur classique.

\subsection{REMPLACEMENT DES MEMBRANES}

La durée de vie des membranes dépend fortement des conditions d'utilisation. Etant donné le peu de recul dont nous disposons à ce jour, il est très difficile d'établir une durée de vie moyenne des membranes synthétiques. Cependant, dans toutes nos études de faisabilité, le coût du remplacement se base sur la durée de la garantie offerte par le constructeur, ce qui est une option pessimiste.

\section{Etude de cas}

La plupart des projets mettent en concurrence évaporation et osmose inverse. C'est le cas des deux projets analysés ci-après, qui pour des taux de concentration finale différents (12 et $24 \%$ S) présentent le bilan énergétique comparatif des deux solutions.

\subsection{Préconcentration de lactosérum de 6 A $12 \%$ MS}

Il s'agit d'augmenter la capacité de traitement et de réduire la consommation énergétique d'un évaporateur existant.

Le matériel existant permet la concentration de 6 à $40 \%$ MS de 10000 litres de lactosérum par heure. La consommation spécifique de vapeur est de 0,29 tonne par tonne d'eau évaporée. En ce qui concerne l'électricité, la puissance appelée est de $45 \mathrm{~kW}$.

La capacité de traitement doit passer de 10 à 15000 litres par heure, la durée annuelle de fonctionnement est de $4000 \mathrm{~h}$. Deux solutions ont été envisagées (cf tab. 1) :

- Ajout d'un quatrième effet à l'évaporateur actuel. La consommation spécifique de vapeur passe à 0,170 tonne par tonne d'eau évaporée. La consommation d'électricité ne varie pas.

- Préconcentration par osmose inverse de 6 à $12 \%$ MS en amont de l'évaporateur existant.
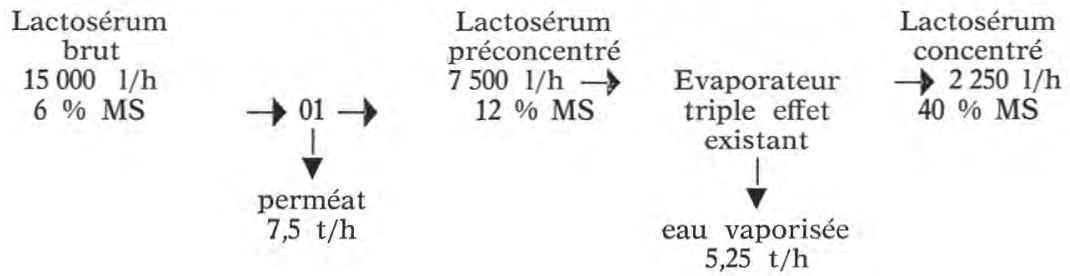
TABLEAU 1

Concentration de lactosérum de 6 à $40 \%$ M.S

Bilan énergétique comparatif

\begin{tabular}{|c|c|c|c|c|}
\hline \multirow[b]{2}{*}{$\begin{array}{l}\text { Consommation de vapeur } \\
\text { - spécifique (tonne par tonne d'eau éva- } \\
\text { porée) } \\
\text { - horaire (tonne par heure) } \\
\text { - annuelle ( } 10^{3} \text { tonne par an) }\end{array}$} & $\begin{array}{l}\text { Evaporateur } \\
\text { quadruple effet } \\
\text { avec thermo- } \\
\text { compression } \\
6 \rightarrow 40 \% \text { M.S }\end{array}$ & $\begin{aligned} & \text { Osmose } \\
& \text { inverse }\end{aligned}$ & $\begin{array}{l}\text { Evaporateur } \\
\text { triple effet } \\
\text { avec thermo- } \\
\text { compression } \\
12 \rightarrow 40 \% \text { M.S }\end{array}$ & $\begin{array}{l}\text { Osmose } \\
\text { inverse } \\
\quad+ \\
\text { évaporateur } \\
6 \rightarrow 40 \% \text { M.S }\end{array}$ \\
\hline & $\begin{array}{l}0,17 \\
2,17 \\
8,67\end{array}$ & $\begin{array}{l}0,003 \\
0,02 \\
0,09\end{array}$ & $\begin{array}{l}0,29 \\
1,52 \\
6,09\end{array}$ & $\begin{array}{l}\overline{1,54} \\
6,18\end{array}$ \\
\hline $\begin{array}{l}\text { Consommation d'électricité } \\
\text { - spécifique (kWh par tonne d'eau éva- } \\
\text { porée) } \\
\text { - horaire (kWh par heure) } \\
\text { - annuelle (MWh par an) }\end{array}$ & $\begin{array}{l}3,5 \\
45 \\
180\end{array}$ & $\begin{array}{c}5 \\
37,5 \\
150\end{array}$ & $\begin{array}{r}\overline{45} \\
180\end{array}$ & $\overline{82,5}$ \\
\hline $\begin{array}{l}\text { Consommation annuelle d'énergie primaire } \\
\text { (tep/an) } \\
\text { (1 tep }=10000 \text { th) } \\
\text { - vapeur }(740 \text { th } / \mathrm{t}) * \\
\text { - électricité }(2,5 \text { th } / \mathrm{kWh}) \\
\text { - total }\end{array}$ & $\begin{array}{l}641,6 \\
45 \\
686,6\end{array}$ & $\begin{array}{r}6,7 \\
37,5 \\
44,2\end{array}$ & $\begin{array}{l}450,7 \\
45 \\
495,7\end{array}$ & $\begin{array}{r}457,4 \\
82,5 \\
539,9\end{array}$ \\
\hline $\begin{array}{l}\text { Economie annuelle d'énergie primaire } \\
\text { (tep/an) }\end{array}$ & & & & 147 \\
\hline $\begin{array}{l}\text { * Vapeur sous } 8 \text { bars; retour des conde } \\
\text { distribution de vapeur de } 81 \% \text { environ : }\end{array}$ & $\begin{array}{l}\text { sats à la chau } \\
\frac{660-60}{0,81}=74\end{array}$ & à $60^{\circ} \mathrm{C} \mathrm{e}$ & ; rendement & roducion et \\
\hline
\end{tabular}


L'installation comprend un seul étage. La surface totale de membranes est de $291 \mathrm{~m}^{2}$, soit un débit moyen de perméat de 25,8 litres par heure et $\mathrm{m}^{2}$ de membranes. La consommation spécifique d'électricité est de $5 \mathrm{kWh}$ par tonne de perméat ou d'eau éliminée. Un préchauffage des solutions de nettoyage est nécessaire ; il correspond à 0,003 tonne de vapeur par tonne d'eau éliminée.

La comparaison de ces deux solutions détermine le coefficient de substitution $\gamma$ au niveau de l'appareil :

$$
\begin{aligned}
\gamma & =\frac{(0,170-0,003) \mathrm{t} \text { de vapeur/t d'eau éliminée } \times 600 \mathrm{th} / \mathrm{t} \text { de vapeur }}{(5-3,5) \mathrm{kWh}} \\
\gamma & =66,8 \mathrm{th} / \mathrm{kWh} \quad
\end{aligned}
$$

Compte tenu d'un rendement de production et de distribution de vapeur de 0,81 , le coefficient de substitution $f_{\text {u }}$ au niveau de l'usine s'établit à :

$$
\Gamma_{u}=\frac{66,8}{0,81}=82,5 \mathrm{th} / \mathrm{kWh} .
$$

Ainsi, pour la plage de préconcentration de 6 à $12 \%$ MS, l'osmose inverse conduit à une économie annuelle d'énergie primaire de $360^{*}$ tep par rapport à l'ajout d'un quatrième effet.

Pour l'ensemble de la concentration de 6 à $40 \%$ MS, l'association osmose inverse et évaporateur triple effet existant permet de dégager une économie annuelle de 147 tep.

\subsection{Préconcentration de lactosérum de 5,2 a $24 \%$ MS}

Il s'agit de réduire la consommation énergétique globale d'un ensemble d'unités de concentration en mettant en place une nouvelle installation très performante et en diminuant la quantité de lactosérum à transporter.

La capacité de traitement est fixée à 12000 litres de lactosérum par heure. L'installation fonctionne $5000 \mathrm{~h}$ par an. Deux solutions ont été envisagées (cf tab. 2) :

- Evaporateur triple effet avec thermocompression concentrant de 5,2 à $32 \%$ MS. La capacité évaporatoire est de 10 tonnes d'eau par heure. La consommation spécifique de vapeur est de 0,175 tonne par tonne d'eau évaporée. La puissance électrique appelée est de $50 \mathrm{~kW}$.

- Osmose inverse concentrant de 5,2 à $24 \%$ MS.

La capacité d'élimination d'eau est de 9,4 tonnes d'eau par heure. L'installation comprend 4 étages. La surface totale de membranes

* 360 tep $=\frac{686,6 \times 7,5}{12,75}-44,2$. 


\section{TABLEAU 2}

Concentration de lactosérum de 5,2 à $24 \%$ MS

Bilan énergétique comparatif

\begin{tabular}{|c|c|c|}
\hline & $\begin{array}{l}\text { Evaporateur triple effet } \\
\text { avec thermocompression }\end{array}$ & Osmose inverse \\
\hline $\begin{array}{l}\text { Consommation de vapeur } \\
\text { - spécifique (tonne par tonne d'eau évaporée) } \\
\text { - horaire (tonne par heure) } \\
\left.\text { - annuelle ( } 10^{3} \text { tonne par an }\right)\end{array}$ & $\begin{array}{l}0,175 \\
1,65 \\
8,23\end{array}$ & - \\
\hline $\begin{array}{l}\text { Consommation d'électricité } \\
\text { - spécifique (kWh par tonne d'eau évaporée ou } \\
\text { extraite) } \\
\text { - horaire (kWh par heure) } \\
\text { - annuelle (MWh par an) }\end{array}$ & $\begin{array}{r}5,5 \\
51,7 \\
258,5\end{array}$ & $\begin{array}{r}7,7 \\
72,4 \\
357,7\end{array}$ \\
\hline $\begin{array}{l}\text { Consommation annuelle d'énergie primaire (tep/an) } \\
\text { (1 tep }=10000 \mathrm{th}) \\
\text { 二 vapeur }(740 \mathrm{th} / \mathrm{t})^{*} \\
\text { - électricité }(2,5 \mathrm{th} / \mathrm{kWh}) \\
\text { total }\end{array}$ & $\begin{array}{r}609 \\
64,6 \\
673,6\end{array}$ & $\begin{array}{l}89,4 \\
89,0\end{array}$ \\
\hline Economie annuelle d'énergie primaire (tep/an) & & 585 \\
\hline
\end{tabular}


est de $437 \mathrm{~m}^{2}$, soit un débit moyen de perméat de 21,5 litres par heure et $\mathrm{m}^{2}$. La consommation spécifique d'électricité est de $7,7 \mathrm{kWh}$ par tonne d'eau éliminée, soit une puissance de $72 \mathrm{~kW}$.

Ces différents éléments permettent de calculer le coefficient de substitution $\gamma$ au niveau de l'appareil :

$0,175 \mathrm{t}$ de vapeur/t d'eau évaporée $\times 600 \mathrm{th} / \mathrm{t}$ de vapeur

$$
\begin{aligned}
& \gamma=\frac{}{\gamma}=38,9 \mathrm{th} / \mathrm{kWh} . \quad(7,7-5) \mathrm{kWh} \\
& \quad
\end{aligned}
$$

$\mathrm{Au}$ niveau de l'usine, le coefficient de substitution $\Upsilon_{\mathrm{u}}$ est :

$$
i_{u}=\frac{38,9}{0,81}=48 \mathrm{th} / \mathrm{kWh} \text {. }
$$

Pour la comparaison des deux solutions (cf tab. 2) le taux de concentration finale de l'évaporation a été ramené à $24 \%$ MS. Dans ce contexte, l'osmose inverse conduit à une économie annuelle d'énergie primaire de 585 tep.

Aux conditions économiques de mi-1983 (110 F la tonne de vapeur et $0,30 \mathrm{~F}$ le $\mathrm{kWh}$ ), le temps de retour brut du surinvestissement est voisin de un an.

\section{Conclusion}

La préconcentration par osmose inverse est une solution très performante applicable aux unités de concentration.

Le développement de cette technique est assez récent comme le prouve le tableau 3.

Ainsi, début 1984, plus de 20 installations sont en exploitation ou en cours de montage pour le seul secteur laitier. La plupart

TABLEAU 3

Développement de l'osmose inverse dans le secteur laitier

\begin{tabular}{c|c|c|c}
\hline $\begin{array}{c}\text { Année de mise } \\
\text { en service }\end{array}$ & $\begin{array}{c}\text { Nombre } \\
\text { d'installations }\end{array}$ & $\begin{array}{c}\text { Capacité } \\
\text { d'élimination } \\
\text { d'eau }(\mathrm{t} / \mathrm{h})\end{array}$ & $\begin{array}{c}\text { Puissance } \\
\text { installée } \\
(\mathrm{kW})\end{array}$ \\
\hline 1980 & 2 & 6 & 30 \\
1981 & 3 & 16 & 80 \\
1982 & 9 & 55 & 545 \\
1983 & $7 *$ & 62 & 530 \\
\hline
\end{tabular}


préconcentre du lactosérum; quelques-unes sont aussi prévues pour préconcentrer du lait écrémé.

L'avantage énergétique de la préconcentration par osmose inverse ne doit pas masquer le fait qu'une part importante du coût annuel d'exploitation est due au remplacement des membranes. Aux capacités d'élimination d'eau moyennes (de l'ordre de $10 \mathrm{t} / \mathrm{h}$ ), le choix entre osmose inverse et recompression mécanique de vapeur n'est donc pas immédiat, compte tenu de niveau d'investissement relativement proches.

* Quatre unités supplémentaires ont été décidées en 1983. Leur réalisation effective a été différée en 1984, du fait de problèmes de financement.

\section{Bibliographie}

Berail (J. F.), Lagabrielle (D.), Richard (Y.) (1981). - La préconcentration du lactosérum. Résultats d'une campagne de mesures. Rapport E.D.F./D.E.R., HP 40/81/345, octobre.

Bimbenet (J. J.), GRAnier (P.). - L'osmose inverse et l'ultrafiltration dans les industries alimentaires. Cahiers du Génie Industriel Alimentaire, $\mathrm{n}^{\circ} 1$.

DRI (D.), Jestin (L.), LeFranc (M.) (1982). - Préconcentration du lactosérum par osmose inverse. Résultats de campagne de mesures. B.S.N. Gervais-Danone, Neufchâtel-en-Bray. Rapport E.D.F./D.E.R., HP 40/82/370, juillet.

Duвоis (G.) (1981). - Préconcentration de lactosérum par osmose inverse. Fromagerie Jean Lincet-Gaye - 51220 Sézanne. Journées C.F.E., La Baule, juin.

Huchon (J.) (1981). - Economie d'énergie en industrie laitière. Le cas de l'opération de concentration. Revue I.A.A., 98 année, juin, $\mathrm{n}^{\circ} 6$.

Huchon (J.), BÉNARD (M.) (1982). - Préconcentration de lactosérum par osmose inverse. Résultats de la campagne de mesures. Fromageries Martin et Collet 08190 Asfeld. Rapport E.D.F./D.E.R., HP 40/82/250, février.

Huchon (J.) (1982). - Concentration de produits laitiers et techniques électriques ( ${ }^{\mathrm{e}}$ partie). Revue Génie Rural, juillet, $\mathrm{n}^{\circ} 7$.

Huchon (J.) (1983). - La concentration des produits laitiers par osmose inverse. Colloque APRIA, Cambrai, septembre.

Marchand (D.), Berail (J. F.), Lagabrielle (D.) (1981). - Préconcentration de lactosérum par osmose inverse. Unicoolait - 57401 Sarrebourg. Rapport E.D.F./ D.E.R., HP 42/81/15, octobre. 